\title{
Serum biopterin - a novel marker for immune activation during pre-diabetes in the BB rat
}

\author{
A.J.Davies ${ }^{1}$, A.J. Bone ${ }^{1}$,T.J.Wilkin ${ }^{1}$, H.Rokos ${ }^{2}$, D.R.Cole ${ }^{1}$ \\ ${ }^{1}$ Endocrine Section, University Medicine, Southampton General Hospital, Southampton, UK \\ ${ }^{2}$ Henning Berlin GmbH, Germany
}

Summary Tetrahydrobiopterin $\left(\mathrm{BH}_{4}\right)$ is a pteridine product which is released by rodent macrophages on activation by cytokines. We have used serial pancreatic biopsy, and measurement of serum biopterin at $30,60,90$ and 120 days in the $\mathrm{BB} / \mathrm{S}$ rat to relate histological change to macrophage activation during the course of prediabetes. Using immunohistochemistry, and an arbitrary scoring system read blind and standardised against day 30, we found that pancreatic MHC class I, MHC class II and infiltrating macrophage staining were upregulated in the $\mathrm{BB} / \mathrm{S}$ diabetes-prone rats $(n=17)$ at day 60 , markedly so at day 90 , and less so at day 120 . Staining for resident pancreatic macrophages remained unchanged throughout in diabetes prone, diabetes resistant and Wistar $(n=28)$ control animals. Serum biopterin fell progressively and identically with age in BB diabetes resistant rats $(n=11)$ and Wistar controls. No change in weight gain or biopterin levels was observed in the biopsied animals. Mean serum biopterin levels in diabetes prone rats (of which 13 of 17 became diabetic at median 85 days) were the same as in diabetes resistant and Wistar rats at days 30,60 and 120 , but showed a striking and highly significant elevation $(p<0.001)$ at day 90 . Although macrophages infiltrate the islet early in prediabetes, the timing of their activation is unknown. The rise in biopterin we observed is a potentially important immunological event which occurred late in the progression of pre-diabetes. This acute terminal event has not been reported previously, and may modify current concepts concerning the tempo of cell destruction during pre-diabetes. [Diabetologia (1994) 37:466-470]

Key words IDDM, biopterin, pre-diabetes, nitric oxide, macrophages, insulitis.
Insulin-dependent diabetes mellitus (IDDM) is thought to result from the destruction of beta cells by activated immunocytes and the cytokines they produce [1]. Studies in the spontaneously diabetic BB rat, a model of IDDM, suggest that macrophages are the earliest infiltrating cell, followed by lymphocytes $[2,3]$.

It has been suggested that the course of beta-cell destruction which leads to IDDM may be linear [4]. The linear model does not, however, explain the seasonal

Received: 6 August 1993

and in revised form: 15 December 1993

Corresponding author: Professor T.J.Wilkin, Department of Medicine, Room 417, Davey Building, University of Plymouth, Plymouth PL4 8AA, UK

Abbreviations: BB, Bio Breeding; IDDM, insulin-dependent diabetes mellitus variation of disease onset [5], the immunological remissions from insulitis which are now well documented, nor the long-term maintenance of islet cell autoimmunity without the development of diabetes [6]. Alternative theories are needed, and one of repeated exacerbation - the "multi-hit" hypothesis - has been suggested [7].

When human bone-marrow derived cells of the monocyte/macrophage series are activated by cytokines, especially interferon- $\gamma$, the enzyme guanosine triphosphate-cyclohydrolase is induced, and large amounts of neopterin are synthesised from guanosine triphosphate as a result [8]. Neopterin is the degradation product of the first intermediate in the biosynthetic pathway from guanosine triphosphate to tetrahydrobiopterin. It is found in the serum and urine of man and considered a valuable marker for the interferon- $\gamma$ activation of macrophages [9]. Elevated levels of 
neopterin have been reported in patients with autoimmune diabetes [10], though not always [11], rheumatoid arthritis [12] and thyroiditis [13]. Neopterin is rarely found in rodents, but high levels of tetrahydrobiopterin, also the result of guanosine triphosphate-cyclohydrolase induction, can be detected in the serum and urine of rats after oxidation to the more stable and more easily measured biopterin. Tetrahydrobiopterin is an obligate co-factor for nitric oxide synthase [14] and nitric oxide is believed to be a key mediator of cell destruction in autoimmune insulitis $[15,16]$.

The aim of this study was to use serum biopterin levels as a marker for monitoring immune response activation during the pre-diabetic prodrome in the $\mathrm{BB}$ rat.

\section{Materials and methods}

Animals. Seventeen diabetic prone and 11 diabetic resistant animals were identified from the Southampton BB rat colony and were studied from 30 to 120 days of age. Twenty-eight control Wistar rats were similarly studied. Mixed venous/arterial blood samples were obtained from the tail vein during serial pancreatic biopsies on or around $30,60,90$ and 120 days of age, and at similar time points in the unbiopsied animals. The $\mathrm{BB}$ rat tolerates multiple pancreatic biopsies without influencing the frequency of diabetes [17]. The BB/S line was derived from a thirtieth generation nucleus supplied by a commercial breeding centre (Mollegaard Ltd., Ejby, Denmark). Successive brother-sister mating has produced the BB/S-diabetic prone and BB/S-diabetic resistant sublines. The Southampton colony is currently in its fourth generation. The colony is maintained in a pathogen-free environment, in which all handlers wear sterilised overalls, a filtered helmet and sterile gloves. The animals are fed on a breeding diet of irradiated rat chow (Bantin and Kingman, Hull, UK) and given free access to sterilised water. Animals removed for serial pancreatic biopsy were not returned to the pathogen-free colony afterwards, and the Wistar controls were not maintained under pathogen-free conditions. Light was provided for all animals on a $14 / 10 \mathrm{~h}$ light/dark cycle with the temperature maintained at approximately $21^{\circ} \mathrm{C}$. The animals were weighed daily and the urine analysed for glucose and ketones using Uristix (Ames and Co., Stoke Poges, UK). If glycosuria was noted, the diagnosis of IDDM was confirmed the same day by a random blood glucose determination ( $>20 \mathrm{mmol} / \mathrm{l}$ ) using BM sticks (Boehringer Mannheim Ltd., East Grinstead, UK). Diabetic rats were maintained on single daily injections of subcutaneous bovine Ultralente, $40 \mathrm{IU} / \mathrm{ml}$ (Novo-Nordisk, Bagsvaerd, Denmark), the dose adjusted according to weight and degree of glycosuria.

Pancreatic biopsies. A modification of the method described by Logothetopoulos et al. [18] was followed, allowing easy removal of a small portion of the splenic pancreas. Anaesthesia was achieved using $2.5 \%$ halothane delivered with oxygen through a mask. In brief, a $2.5 \mathrm{~cm}$ midline incision was made in the abdomen, and the pancreas exposed by exteriorisation of the spleen. A small portion of pancreas was clamped off with artery forceps, an approximately $100 \mathrm{mg}$ biopsy removed with scissors, and haemostasis assured before removal of the clamp. The tissue was immediately snap-frozen in liquid nitrogen. The muscular wall was closed along the linea alba, and the skin closed with wound clips.

Biopterin assay. All material for the competitive radioimmunoassay kit, including a ${ }^{125}$ I-labelled rat biopterin tracer and a highly specific biopterin antiserum, raised in rabbit, were supplied by Henning Berlin GmbH (Germany). A large percentage of biopterin in the body fluids exists as tetrahydrobiopterin and acidic oxidation permits the determination of total biopterin. In brief, a $60 \mu \mathrm{l}$ sample of serum or standard was pipetted into a microfuge tube, to which were added $10 \mu \mathrm{l}$ of freshly prepared oxidising solution. The oxidising solution was produced by adding $1.8 \mathrm{ml}$ of $2 \mathrm{~mol} / 1 \mathrm{HCl}$ to $300 \mathrm{mg}$ of manganese dioxide, with thorough mixing to produce a homogenous suspension. The sample tubes were mixed well and incubated for $1 \mathrm{~h}$, protected from light. The reaction was terminated by adding $300 \mu \mathrm{l}$ of a phosphate buffer, mixed thoroughly and centrifuged at $2000 \mathrm{~g}$ for $5 \mathrm{~min}$. The $\mathrm{pH}$ of the supernatant was confirmed at 6.5 , and retained as oxidised serum for biopterin estimation in the second phase of the assay.

Of the oxidised serum or standard $50 \mu \mathrm{l}$ was pipetted into a $1.9 \mathrm{ml}$ microfuge tube followed by $100 \mu \mathrm{l}$ of biopterin tracer and $100 \mu \mathrm{l}$ of biopterin antiserum. The tubes were sealed and mixed, then incubated for $2.5 \mathrm{~h}$ at room temperature in the dark. Additional tubes were set up in duplicate, first to determine the total amount of labelled biopterin added and, second, to determine the amount of tracer left in the tube in the absence of antibody.

On completion of the incubation, $1 \mathrm{ml}$ of washing solution was added to precipitate the antibody-antigen complex, mixed and then centrifuged for $10 \mathrm{~min}$ at $2000 \mathrm{~g}$. The washing solution disturbed the equilibrium achieved during incubation, thus it was added only to those tubes that could be centrifuged immediately. The remaining tubes were left unwashed until ready for centrifugation. The supernatant was removed by gentle aspiration, and the radioactivity of each tube counted for $120 \mathrm{~s}$ in a gamma counter.

Serial serum dilutions showed parallelism with the standard curve. Two serum samples were spiked with known quantities of biopterin standard, and recovery varied from $86 \%$ to $111 \%$. The inter-assay coefficient of variation for three points on the standard curve varied between 9.2 and $11.2 \%$. The corresponding intra-assay variation varied between 8.0 and $8.5 \%$. The assay sensitivity, based on the point $2.5 \mathrm{SD}$ above the mean result of analysing the zero standard ten times, was $2.35 \mathrm{nmol} / 1$. The mean binding coefficient, based on the maximum bound/total counts added, and measured in ten assay runs was $61.8 \pm 7.17 \%$ (mean $\pm \mathrm{SD}$ ).

Immunohistochemistry. Five $\mu \mathrm{m}$ tissue sections from each block were cut through the full thickness, so as to be representative of the whole biopsy, and every tenth section was placed on a "scan" slide which was later stained for class II expression and used to assess the degree of infiltration in different parts of the tissue. The sections were placed on methanol-cleaned microscope slides, air dried for $1 \mathrm{~h}$ by electric fan and kept at $-20^{\circ} \mathrm{C}$ until use.

A two-step indirect staining method was used to localise antigen. The primary antibody was incubated with the tissue and, after washing, an enzyme-labelled secondary antibody was added. The primary antibodies were all mouse monclonals (Sigma, Poole, Dorset, UK) at dilutions 1/100 (ED1 - infiltrating macrophages [19], 1:150 (ED2 - resident macrophages [19]) and 1:200 (OX6 - MHC class II and OX18 - MHC class I), and the secondary antibodies peroxidase-conjugated rabbit anti-mouse (Sigma) at a dilution of 1:50. The chromagen used was aminoethylcarbazole. Background staining was minimised by diluting the antibodies in phosphate buffered saline containing $10 \%$ normal rabbit serum and $10 \%$ normal sheep serum. A negative control was used for each slide to confirm the absence of nonspecific staining and endogenous enzyme activity. The control lacked the primary antibody. Both primary and secondary antibodies were incubated for $60 \mathrm{~min}$ in a humidified chamber at room temperature. The slides were counter-stained with 
Mayer's haematoxylin for $1 \mathrm{~min}$ to reveal surrounding tissue architecture and "blued" for $5 \mathrm{~min}$ in running tap water.

Seventeen of the Wistar controls were biopsied, two of the $\mathrm{BB}$ diabetes resistant rats and ten of the diabetes prone rats. Cellular infiltration was scored semiquantitatively and applied to sections which were coded and assessed without knowledge of the sample identity. Each biopsy provided a single block, and the sections to be scored were selected in two ways. First, every 10 th 5 - $\mu \mathrm{m}$ section (six to 10 per block) was stained with OX6 (monocytes and lymphocytes), as described, both to identify up to six infiltrated islets per block and at the same time provide a measure of the mean degree of immunocyte infiltration in that particular pancreas. Second, each of the (maximum) six infiltrated islets was serially sectioned, and the degree of ED1, ED2, OX6 and OX18 staining assessed from mean scores of eight consecutive sections. A mean score was calculated for the sections examined from each biopsy, and an average of the means derived for each time point $(30,60,90$ and 120 days) for each group of animals (BB/S-diabetes prone, BB/S-diabetes resistant and Wistar controls).

Class II staining (OX6) was ranked as follows: $0=$ normal OX6 + cell distribution (compared to control Wistar pancreas); $1=$ periductal and perivascular infiltration of OX $6+$ cells; $2=$ peripheral islet \pm periductal/perivascular infiltration of OX6 + cells; $3=$ complete infiltration of the majority of islets with OX6 + cells. Class I MHC staining (OX18) was classified in the following way: $0=$ normal expression (compared to Wistar controls); 1 = increased class I staining on vascular endothelium; 2 = increased class I expression in islets \pm peri-islet tissue; 3 = increased class I expression in a whole pancreatic lobule. Finally, the distribution of ED1 + and ED2 + monocytes was assessed on a scale 0-2:0 = normal sparse distribution (as compared with Wistar controls); 1 = periductal and/or perivascular infiltration; $2=\mathrm{ED} 1+$ or ED2 + cells within islets.

\section{Statistical analysis}

The serum biopterin concentrations for each group were compared using the non-parametric Mann-Whitney test. Correlation coefficients were derived by the method of least squares.

\section{Results}

Blood samples and pancreatic biopsies were obtained from seven $\mathrm{BB} / \mathrm{S}$-diabetes prone rats, nine $\mathrm{BB} / \mathrm{S}$ diabetes resistant rats and 14 Wistar controls. Blood samples alone were obtained in a further 10,2 and 14 rats, respectively. All biopsies and blood sampling were undertaken within $72 \mathrm{~h}$ of the time indicated by the protocol, and those BB animals which were bled, but not biopsied, remained in the pathogen free environment throughout. Thirteen of the $17(76 \%)$ of the $\mathrm{BB} / \mathrm{S}$-diabetes prone animals developed diabetes. The mean age of onset was 85 days, with a range of 74 96 days. Diabetes developed in all the BB/S-diabetes prone animals biopsied. All the $\mathrm{BB} / \mathrm{S}$-diabetes resistant and Wistar control rats, including those biopsied, remained diabetes free. Weight gain and mean serum biopterin levels were unaffected by biopsy, being indistinguishable between biopsied and non-biopsied animals at all time points (data not shown).

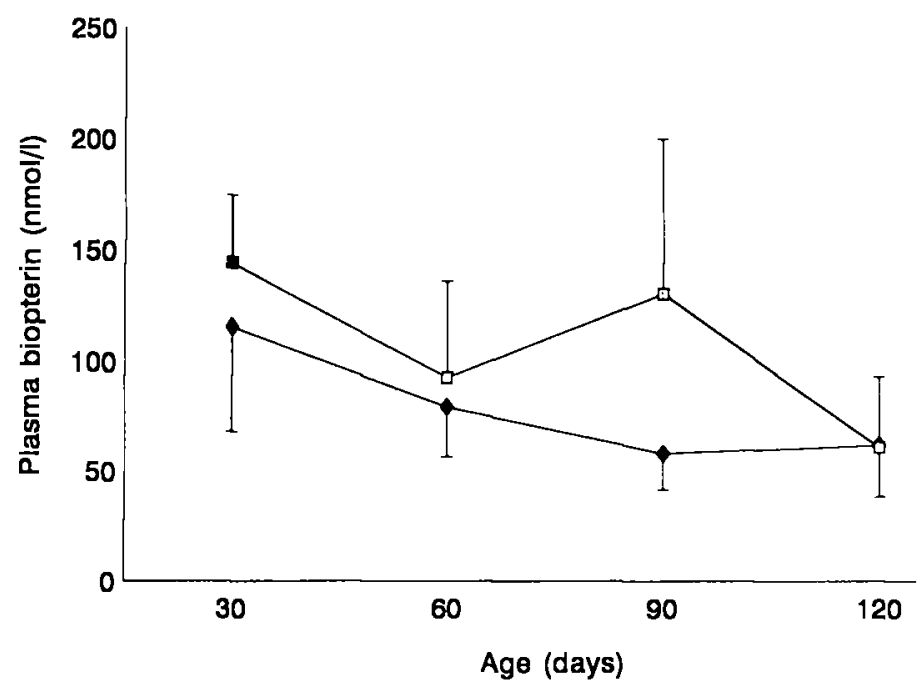

Fig. 1. Mean plasma biopterin concentrations in BB/S-diabetes prone rats $(n=17, \square)$ and Wistar controls $(n=28, \bullet)$

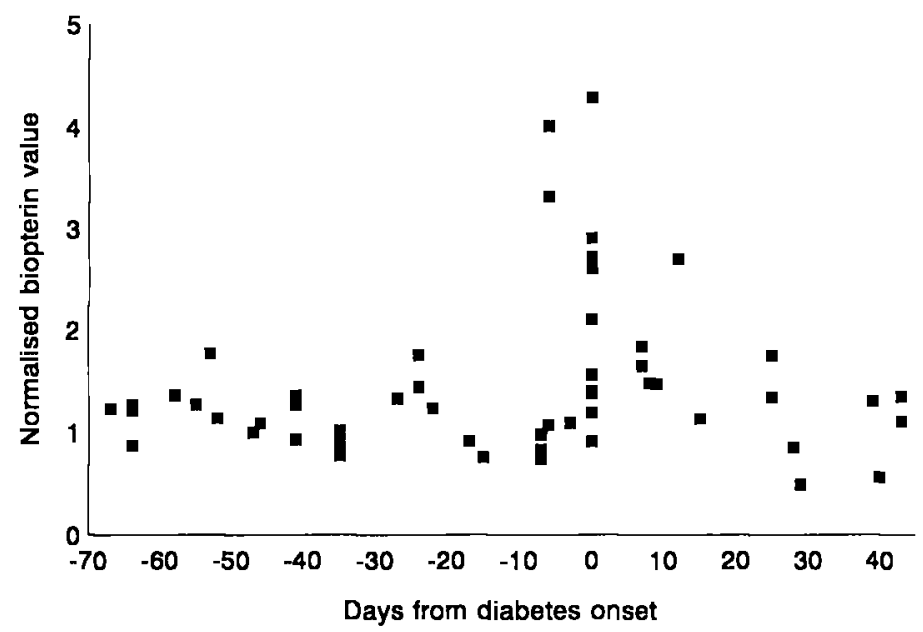

Fig. 2. The plasma biopterin concentrations in BB/S-diabetes prone rats $(n=13)$ corrected at each time point for the mean value in Wistar controls of the same age, and presented relative to the day of diabetes onset

Serum biopterin levels. The serum concentration of biopterin fell progressively with time in the Wistar control animals $(p<0.05)$, and the pattern was similar in the BB/S-diabetes resistant rats (data not shown). The biopterin levels in $\mathrm{BB} / \mathrm{S}$-diabetes prone rats were also similar to those in Wistar controls or in BB/S-diabetes resistant rats at 30,60 and 120 days of age. There was, however, a sharp and highly significant rise $(p<0.001)$ in the $\mathrm{BB} / \mathrm{S}$-diabetes prone rats at 90 days, which was not seen in either the diabetes resistant animals or the controls (Fig. 1). The acuteness of this rise is best illustrated in Figure 2 where the serum biopterin concentration of individual $\mathrm{BB} / \mathrm{S}$-diabetes prone rats is expressed as a ratio of the age-corrected mean biopterin concentration in Wistar controls, relative to the time from diabetes onset. All biopterin data, including that from samples taken on the day of diabetes onset, are included in Figure 2. The serum biopterin ratio between 
Table 1. Immunohistochemistry of $5-\mu \mathrm{m}$ cryosections obtained from serial pancreatic biopsies in $\mathrm{BB} / \mathrm{S}$ diabetes prone rats, using an arbitrary scoring system (see Materials and methods) standardised to day 30

\begin{tabular}{lrrll}
\hline Day & 30 & 60 & 90 & 120 \\
\hline Class I & 1 & 1.7 & 18.9 & 11.9 \\
Class II & 1 & 10.5 & 42 & 32.7 \\
ED1 & 1 & 11.6 & 39.1 & 26.6 \\
\hline
\end{tabular}

$\mathrm{BB} / \mathrm{S}$-diabetes prone and Wistar controls averaged unity from day -70 to day -10 , and from day +10 to day +40 . However, over the period day -10 to day +10 , there was a striking rise in serum biopterin concentrations distributed narrowly around the day of diabetes onset. Three of the 13 animals which became diabetic failed to show a peak at the 90-day measurement, while all but one of the four BB/S-diabetes prone rats in which clinical disease did not develop showed a biopterin peak at this time point. Five diabetes prone rats in which a biopterin peak was demonstrated nevertheless had normal levels on the day diabetes was first detected. The peaks were always within a few days and always at or before - the onset of disease.

Immunohistochemistry. Table 1 shows the mean scores for class II MHC (OX6), class I MHC (OX18), ED1 and ED2 expression at each time point. With the exception of ED2, there was a progressive and rapid increase to day 90, with a significant fall by day 120 . There were close correlations between the mean scores for OX6, OX18 and ED1 staining within pancreata: OX6 vs OX18 $(r=0.80, p<0.01)$; OX6 vs ED1 $(r=0.84, p<0.01)$. There was no correlation between degrees of ED1 + or MHC staining and serum biopterin levels.

Occasional perivascular OX6 + cell cuffing was observed in the day 90 biopsies from $\mathrm{BB} / \mathrm{S}$-diabetes resistant animals, although the islets remained clear of lymphocytic infiltration. Mild vascular endothelial upregulation of class I MHC staining was observed which did not affect islets. ED1 staining was not found in the $\mathrm{BB} / \mathrm{S}$-diabetes resistant pancreata. ED2 staining was found scattered in the interlobular connective tissue of the exocrine, but not endocrine, pancreas and remained unchanged throughout in diabetes prone, diabetes resistant and Wistar animals.

\section{Discussion}

We have used the technique of serial pancreatic biopsy and tail blood sampling to document the relationship between islet cell infiltration and serum biopterin levels in diabetes-prone $\mathrm{BB} / \mathrm{S}$ rats, diabetes-resistant $\mathrm{BB} / \mathrm{S}$ rats and Wistar controls. We have confirmed previous reports of a sequence of events involving $\mathrm{MHC}$ class I upregulation, ED1 + /OX6 + macrophage infil- tration followed in time by other ED1 - /OX6 + cells indicative of lymphocytic infiltration [2].

We had anticipated that macrophage activity, as measured in serum biopterin levels, would reflect the cellular infiltration, but this was not observed. Instead, there was a brisk rise in serum biopterin levels only at diabetes onset, the source of which, although likely to be macrophage and/or fibroblast, has yet to be localised. Biopterin production is induced by interferon$\gamma$ acting on the enzyme guanosine triphosphate-cyclohydrolase I [8]. It seems unlikely that the burst of biopterin was secondary to the onset of diabetes, because it was observed in three of the four BB/S-diabetes prone animals in which diabetes did not develop. Perhaps the damage induced in these three animals was insufficient to cause clinical disease. A better experimental design for the present study would have been to obtain blood samples at more frequent intervals around IDDM onset, as some of the diabetes prone rats became diabetic many days after the last sample was taken.

Serial biopsy had no adverse effect on weight gain, and none of the biopsied diabetes resistant or Wistar animals developed diabetes. There was no evidence that biopsy influences pancreatic histology; the ED2 staining in diabetes prone, as well as in diabetes resistant and Wistar rats, remained unchanged throughout, suggesting that the infiltration seen only in the diabetes prone strain occurred independently of pancreatic surgery. Macrophages have been implicated by several investigators as a source of interleukin-1 which can destroy beta cells $[20,21]$. The data have been obtained largely in vitro, and little is known about the time course of interleukin- 1 secretion by macrophages during the pre-diabetic period. It has proved extremely difficult to demonstrate interleukin- 1 by immunohistochemistry in the pre-diabetic pancreas. Macrophages clearly have an important role in vivo, however, as the onset of diabetes can be prevented by the intraperitoneal administration of silica which is phagocytosed by macrophages and renders them inert [22].

In choosing to measure biopterin as a marker of immune response activation, we identified a substance which is liberated into the circulation in vivo in rodents. In man, the corresponding compound neopterin has been validated as a marker of autoimmune reactivity [10-12]. The assay we used was easy to perform and was satisfactorily validated in our laboratory. Its sensitivity $(2.4 \mathrm{nmol} / \mathrm{l})$ was more than adequate to measure serum biopterin in all the samples tested.

It has been suggested that pre-diabetes is a process of slow attrition of cells which occurs, in the human, over a period of months or years [4]. If serum biopterin reflects the activation of macrophages, either generally or those local to the islets, our observations may suggest a different tempo to the progression of pre-diabetes, at least in the BB rat. It would appear that an important immunological event occurs at the time of diabetes onset only in the diabetes-susceptible strain. The event 
does not occur earlier in pre-diabetes and is not always followed by the onset of disease, but diabetes occurred (apparently) without it in only 3 of 13 animals and this may have been due to poor timing in the collection of the blood samples.

We now need to identify the source of the biopterin we measured and to establish that biopterin release immediately precedes every case of diabetes. This report may be the first to document the functional rather than anatomical events of insulitis, and the hypothesis we have outlined is testable.

Acknowledgements. AD was in receipt of a Wolffson Fellowship. TJW was generously supported by the Wellcome Trust, DC by the Wessex Medical Trust and AJB by Eli Lilly Co. Ltd. Our thanks to Mrs. W.Couper and Mrs. E. Baxter for preparing the manuscript.

\section{References}

1. Mandrup-Poulsen T, Helquist S, Molvig J, Wogensen LD, Nerup J (1989) Cytokines as immune effector molecules in autoimmune endocrine diseases, with special reference to insulin-dependent diabetes mellitus. Autoimmunity 4: 191218

2. Walker R, Bone AJ, Cooke A, Baird JD (1988) Distinct macrophage sub-populations in pancreases of pre-diabetic $\mathrm{BB} / \mathrm{E}$ rats: possible role for macrophages in the pathogenesis of IDDM. Diabetes 37: 1301-1304

3. Kolb-Bachofen V, Kolb H (1989) A role for macrophages in the pathogenesis of type 1 diabetes. Autoimmunity 3: 145154

4. Srikanta S, Ganda OP, Gleason RE, Jackson RA, Soeldner JS, Eisenbarth GS (1984) Pre-type 1 diabetes: linear loss of cell response to intravenous glucose. Diabetes 33: 717-720

5. Gamble DR (1969) Seasonal incidence of diabetes mellitus. BMJ 3: 631-633

6. McCulloch DK, Klaff LJ, Kohn SE et al. (1990) Non-progression of subclinical beta-cell dysfunction among first degree relatives of IDDM patients. Diabetes 39: 549-556

7. Karjalainen J, Martin JM, Knip M et al. (1992) Evidence for a bovine albumin peptide as a candidate trigger of type 1 diabetes. N Eng J Med 327: 302-307

8. Huber Ch, Batchelor JR, Fuchs D et al. (1984) Immune-response associated production of neopterin. J Exp Med 160: 310-316

9. Wachter H, Fuchs D, Hansen A, Reibnegger G, Werner ER (1989) Neopterin as a marker for activation of cellular im- munity: immunologic basis and clinical application. Adv Clin Chem 27: 81-141

10. Manna R, Gambassi G, Papa G (1987) Urinary neopterin levels of insulin dependent diabetes mellitus (IDDM) at onset. In: Pfleiderer W, Wachter H, Blair JA (eds) Biochem Clin Aspects Pteridines. de Gruyter, NY, pp 353-358

11. Bottazzo GF, Bonifacio E, Wagner R, al-Sakkaf L, Dean BM, Mirakian R (1990) On the pathogenesis of type 1 (insulin-dependent) diabetes mellitus: facts, areas still under development and new perspectives. Klin Wochenschr 68 [Suppl 21]: 26-27

12. Maerker-Alzer G, Diemer O, Struemper R, Rohe M (1986) Neopterin production in inflamed knee joints: high levels in synovial fluids. Rheumatol Int 6: 151-154

13. Fuchs D, Hansen A, Reibnegger G, Werner ER, Dierich MP, Wachter H (1988) Neopterin as a marker for activated cellmediated immunity. Immunol Today 9: 150-155

14. Tayeh MA, Marletta MA (1989) Macrophage oxidation of L-arginine to nitric oxide, nitrite and nitrate. J Biol Chem 264: 19654-19658

15. Corbett JA, McDaniel ML (1992) Does nitric oxide mediate autoimmune destruction of cells? Diabetes 41: 897-903

16. Kolb H, Kolb-Bachofen V (1992) Type 1 (insulin-dependent) diabetes mellitus and nitric oxide. Diabetologia 35:796

17. Bone AJ, Walker R, Varey AM, Cooke A, Baird JD (1990) Effect of cyclosporine on pancreatic events and development of diabetes in BB/E rats. Diabetes 39: 508-514

18. Logothetopoulos J, Valequette N, Madura E, Cuet D (1984) The onset and progression of pancreatic insulitis in the overt, spontaneously diabetic, young adult BB rat studied by pancreatic biopsy. Diabetes 33: 33-36

19. Dijkstra CD, Dopp EA, Joling P, Kraal G (1985) The heterogeneity of mononuclear phagocytes in lymphoid organs: distinct macrophage subpopulations in the rat recognised by monoclonal antibodies ED1, ED2 and ED3. Immunology 54: 589-599

20. Kroncke KD, Kolb-Bachofen V, Berschick B, Burkart V, Kolb H (1991) Activated macrophages kill pancreatic syngeneic islet cells via arginine-dependent nitric oxide generation. Biochem Biophys Res Commun 175: 752-758

21. Wogensen LD, Reimers J, Nerup J et al. (1992) Repetitive invitro treatment with human recombinant interleukin-1 modifies beta cell function in normal rats. Diabetologia 35: 331-339

22. Hanenberg H, Kolb-Bachofen V, Kantwerk-Funke G, Kolb $H$ (1989) Macrophage infiltration precedes, and is a prerequisite for, lymphocytic insulitis in pancreatic islets of prediabetic BB rats. Diabetologia 32: 126-134 